\title{
Characteristics of Type 2 Diabetes Patients and Their Association with the Metabolic Syndrome and Cardiovascular Risk Factors at Thika Level Five Hospital in Kenya
}

\author{
Thuita Ann Watetu*, Kiage Nyanchama Beatrice, Onyango Arnold N., Makokha Anselimo \\ Department of Human Nutrition Sciences, School of Food and Nutrition Sciences (SoFNuS), Jomo Kenyatta University of Agriculture and \\ Technology, Nairobi, Kenya
}

Email address:

awambugu78@gmail.com (Thuita A. W.), ann.thuita@jkuat.ac.ke (Thuita A. W.), bettynyanchama@yahoo.com (Kiage N. B.), arnoldonyango@jkuat.ac.ke (Onyango A. N.), amakokha@jkuat.ac.ke (Makokha A.)

${ }^{*}$ Corresponding author

\section{To cite this article:}

Thuita Ann Watetu, Kiage Nyanchama Beatrice, Onyango Arnold N., Makokha Anselimo. Characteristics of Type 2 Diabetes Patients and Their Association with the Metabolic Syndrome and Cardiovascular Risk Factors at Thika Level Five Hospital in Kenya. International Journal of Diabetes and Endocrinology. Vol. 4, No. 2, 2019, pp. 35-48. doi: 10.11648/j.ijde.20190402.11

Received: January 25, 2019; Accepted: March 20, 2019; Published: May 10, 2019

\begin{abstract}
Type 2 diabetes is a public health problem and one of the most common life threatening conditions globally, due to its related complications that are usually very costly to treat, with increasing number of people being diagnosed with this condition each year. Presence of the metabolic syndrome (MetS) and cardiovascular risks in Type 2 Diabetes patients increases the risk to complications. The objective of this study was to determine characteristics of type 2 diabetes patients and their association with MetS and associated cardiovascular risk. The study employed a cross section design method. Subjects with Type 2 Diabetes were recruited for the study from Thika Level Five Hospital in Kenya. Socio-demographic, clinical and lifestyle data were obtained using questionnaires. The nutrition status was determined by anthropometry. Other laboratory parameters that were determined included total cholesterol (TC), high density cholesterol (HDL-c) and low density cholesterol (LDL-c), triglyceride (TG), fasting blood glucose (FBG), glycated haemoglobin (HbA1C), and blood pressure (BP). Overall 153 (40.5\% men and 59.5\% women) Type 2 diabetic patients aged 20-79 years were included in the study. The overall mean age of patients was 56.07 years. The prevalence of the metabolic syndrome was $86.3 \%$ as per WHO criteria. The MetS components were elevated waist circumference (WC, 90.8\%), increased waist hip ratio (WHR, 86.9\%), elevated blood pressure $(65.7 \% \& 72.5 \%)$ and elevated triglycerides $(64.8 \%)$. The prevalence of occurrence of the components of the MetS was not significantly different among male and female patients except for WC, BMI and reduced serum HDL-C where women were at a significantly higher risk than men $(\mathrm{P} \leq 0.00$.). The current study showed that income was associated with elevated diastolic blood pressure (BP), secondary education and years lived with diabetes were associated with elevated TG, while occupation showed some association with high WHR. Additionally Gender, marital status and type of residence were associated with elevated HDL while education, family history of diabetes and alcohol intake was associated with obesity. The prevalence of the MetS and associated cardiovascular risk among Type 2 Diabetes patients was high and similar among males and females. Enhanced surveillance on Mets and associated cardiovascular risk in Type 2 Diabetes in addition to application of preventive measures are critical in order to reduce the risk of macro vascular complications as well as increased cardiovascular risks in Type 2 Diabetes patients.
\end{abstract}

Keywords: Metabolic Syndrome, Type 2 Diabetes, Cardiovascular Risk and Patient Characteristic 


\section{Introduction}

Type 2 Diabetes is a group of metabolic disorders of multiple etiologies characterized by chronic hyperglycemia [1] It is further characterized by disturbances of carbohydrate, fat and protein metabolism as a result of insulin resistance and relative insulin deficiency; both of which may be present at the time that diabetes becomes clinically manifested [2-4]. It is a major risk for cardiovascular diseases (CVD) and metabolic syndrome (MetS) [5]. It is a public health problem and one of the most common life threatening conditions globally, due to its related complications that are usually very costly to treat, with more and more people living with the condition each year $[2,4,6,7]$. It is the fourth leading cause of death in most developed countries [6-8]. It is also the main cause of morbidity with a fast growing incidence due to demographic transition and changes in the population's lifestyle [4, 6-8]. Typically, this type of diabetes is diagnosed in people aged 20 years or older [4, 7]. Increasingly, however, it is being diagnosed in younger peoples too $[4,7]$. Poor glycemic control in addition to presence of MetS in Type 2 Diabetes patients worsens the condition further due to related complications and increases the risk for development of CVD $[2,9,10]$.

The MetS comprises a complex of interrelated risk factors that include abdominal obesity, dyslipidemia (low level of high density lipoprotein cholesterol (HDL-C) and /or high triglycerides level (TG)/low density lipoproteins (LDL-c), hypertension and hyperglycemia as a result of insulin resistance [11-14]. It increase the risk of developing Type 2 Diabetes by fivefold and CVD by two folds [13, 15]. These risks; MetS, Type 2 Diabetes and CVD risk factors are therefore closely interrelated (14-17). Studies conducted on Type 2 Diabetes patients have shown high prevalence of MetS and associated risks [10, 14, 16-19] This pose a greater risk to microvascular and macrovascular complications in addition to development of CVD [6, 7]. Furthermore, patient characteristics (socio demographic, lifestyle and clinical characteristic) have also been shown to be a strong predictor of developing MetS, Type 2 Diabetes and occurrence and progression of CVD [14, 18, 20, 21]. In Kenya studies on MetS status and cardiovascular risk factors have been determined in the general population in some regions and have shown a high prevalence of $>50 \%[16,22]$. However, there is very limited information on MetS prevalence and cardiovascular risk factors among Type 2 Diabetes patients. Therefore, the aim of this study was to determine the association of Type 2 diabetes patient characteristics with MetS and cardiovascular risk factors.

\section{Methodology}

\subsection{Study Design}

The study applied a cross section design to find out the characteristics of Type 2 Diabetes patients and their association with the MetS and associated CVD risk factors.

\subsection{Study Setting}

The study was conducted at Thika Level 5 Hospital
(TL5H), Kiambu County, Kenya on Type 2 Diabetes patients attending the Diabetes Comprehensive Centre (DCC).

\subsection{Population}

\subsubsection{Study Participants}

The Study participants were men and women aged 20-79 years with Type 2 Diabetes attending care at the DCC in $\mathrm{TL} 5 \mathrm{H}$. They were recruited during their monthly clinic attendance while waiting to see a health professional. Recruitment was done over a period of two months from August 2016 to October 2016.

\subsubsection{Inclusion Criteria}

Patient suffering from Type 2 diabetes aged between 20-79 years with regular attendance at the DCC who signed an informed consent and were willing to participate in the study were included.

\subsubsection{Exclusion Criteria}

Type 2 Diabetes patients with complications which included renal failure, congestive heart failure (CCF), and stroke were excluded from the study. Pregnant women and HIV patients with Type 2 diabetes were also excluded.

\subsection{Sample Size}

A target sample size of 153 patients calculated using the formula by Armitage et al., [23] and Lwanga and Lemeshow [24] was used for the study.

\subsection{Data Collection}

\subsubsection{Baseline Data (Social Demographic, Anthropometry, Clinical and Physical Activity)}

The demographic data and medical history were obtained using structured questionnaires. Anthropometric measurements that included weight, height, waist circumference and hip circumference were also done. Height and weight was measured using standard protocol with the participant wearing light clothing and no shoes [25]. Weight was measured to the nearest $0.1 \mathrm{~kg}$ using a pre-calibrated Seca scale (SECA, Hamburg, Germany model no. 786/2021994), while height was measured to the nearest $0.1 \mathrm{~cm}$ using a stadiometer attached to the scale as per CDC protocol [25]. The participants were requested to stand straight, with their body weight evenly distributed, both feet flat on the platform with the heels together and toes apart, the back of the head, shoulder blades, buttocks and heels in contact with the stadiometer backboard plus their heads in the Frankfort horizontal plane. The stadiometer head piece was then lowered so that it rested firmly on top of the participant's head, with sufficient pressure to compress the hair. The participants were then requested to take a deep breath and the reading recorded while the patient released the breath. Two readings of each (weight and height) were taken and the average recorded. Body mass index (BMI) was then calculated as weight (kilograms)/height (meters) ${ }^{2}$ and classified as per WHO classification [26]. The waist circumference was measured midway between the lower rib margin and the iliac crest with an anthropometric tape while hip circumference was measured as 
the maximal circumference around the buttocks posteriorly and pubic symphysis anteriorly as per WHO protocol [27]. The waist circumference and hip circumference was measured twice to the nearest $0.5 \mathrm{~cm}$ while the participants were standing relaxed with their feet apart and arms on the sides. If the variation between these two measurements was greater than $2 \mathrm{~cm}$, a third measurement was taken and the mean calculated using the two closest measurements. Blood pressure was measured by trained nurses on left arms with a Spengler digital sphygmomanometer (model: Autortensio ${ }^{\circledR}$ noSPG440), while the participants were in a seated position and the arm supported at heart level. There was at least a 10-minute rest period before the measurement. Two measurements were taken from all the participants at 2-min intervals, and the mean of the measurements used as the final measurement. Readings from the blood pressure machine were recorded to the nearest $2 \mathrm{~mm} \mathrm{Hg}$.

Information on physical activity pattern was collected using a modified WHO designed physical activity questionnaire. [28]. Accordingly, the physical activity level of the participants was categorized using metabolic equivalent (MET) as per the WHO classification [29]. This classification included light physical activities that included light house work job accumulating a MET minutes per week of $<600 \mathrm{MET}$ minute/week; moderate physical activities that included the routine productive activities of an electrician, mechanics, jogging, walking accumulating MET minutes per week of 600-1499 and heavy/vigorous physical activities that included productive activities of nonmechanized agriculture, dance, sports, aerobics digging accumulating MET minute per week of $\geq 1500$. Participants who participated in moderate activities and accumulated $\geq 3000$ MET minutes were also considered in the vigorous physical activity category. The average energy expenditure and duration of total physical activity per week was calculated from the questionnaire and recorded in MET minute week

\subsubsection{Laboratory Assay}

Blood samples were collected from each participant while in a seated position after fasting for at least $8-12 \mathrm{hrs}$. Within 1 hour of blood collection, the samples were centrifuged and separated. Lipid profile (total cholesterol, triglycerides, high density lipoprotein cholesterol [HDL-C] and blood glucose estimation were determined using enzymatic method. Level of serum TG was determined using Glycerol Phosphate Oxidase Peroxidase GPO/POD, endpoint method [30], total cholesterol (TC) using Cholesterol Oxidase Peroxidase (CHOD-POD), end point method [31, 32] and high density lipoprotein (HDL-c) using Phosphotungstic Acid, end Point method [33]). Serum low density cholesterol (LDL-C) was calculated using the Friedwald's formula (LDL-cholesterol $(\mathrm{mmol} / \mathrm{l})=$ Total cholesterol $-(\mathrm{HDL}+$ triglycerides/2.181) [34]. All parameters were read on a spectrophotometer (Dilui $240 \mathrm{~T}$ autoanalyzer) at 510nm Glycated hemoglobin (HbA1c) was determined by Biorad D-10 hemoglobin testing system an automated analyzer, intended for percent determination of HbA1c in human blood using high-performance liquid chromatography [35]. Fasting plasma glucose was determined by glucose oxidase method [36].

\subsubsection{Metabolic Syndrome Diagnosis Criteria}

Metabolic syndrome in the study was defined according to WHO criteria [35] This criteria requires the presence of diabetes mellitus, impaired glucose tolerance or insulin resistance, and any two of the following:(1) body mass index $(B M I) \geq 30 \mathrm{~kg} / \mathrm{m}^{2}$ and/or waist-to-hip ratio $>0.90$ (male), $>0.85$ (female); (2) blood pressure $\geq 140 / \geq 90 \mathrm{mmHg}$ or on hypertension medication; and (3) triglyceride $\geq$ $1.7 \mathrm{mmol} / \mathrm{L}$ and $/$ or HDL-C $<0.91 \mathrm{mmol} / \mathrm{L}$ (male), $<1.01 \mathrm{mmol} / \mathrm{L}$ (female).

\subsubsection{Classification of Other CVD Risk Factors}

Glycemic status control was categorized as good glycemic control if $\mathrm{HbA} 1 \mathrm{c}$ is $<7 \%$ and poor control $\mathrm{HbA} 1 \mathrm{c}$ is $>7 \%$ as per the American Diabetes Association (ADA) guidelines [1, 37]. Elevated waist circumference was considered as waist circumference of $\geq 94 \mathrm{~cm}$ in males and $\geq 80 \mathrm{~cm}$ in females) [13] and BMI was categorized as obese $>30 \mathrm{~kg} / \mathrm{m}^{2}$ and non-obese $<30 \mathrm{~kg} / \mathrm{m}^{2}$ [26] Elevated blood pressure was considered for participants with systolic/diastolic pressure of 130/80 $\mathrm{mmHg}$ or those already using hypertensive drugs [13, 38]. Classification of lipid profiles was done as described by the ADA [1, 37] and American Association of Clinical Endocrinologists and American College Of Endocrinology (AACE-ACE) [39, 40]. These include elevated triglyceride level $\geq 1.7 \mathrm{mmol} / \mathrm{l}$ and/or the use of triglyceride-lowering drugs), reduced HDL cholesterol $(<1.0 \mathrm{mmo} / 1$ in males and $<1.3 \mathrm{mmol} / \mathrm{l}$ in females, elevated LDL cholesterol $(>2.6 \mathrm{mmol} / \mathrm{l})$ and elevated total cholesterol $(>5.2 \mathrm{mmol} / \mathrm{l})[39,40]$.

\subsection{Statistical Analysis}

Statistical analysis was performed using Statistical Package for Social Sciences (SPSS, version 20). Data was presented using mean \pm S.D. for continuous variables and proportions for categorical variables. Categorical variables were compared using Chi-Square test. Independent t-test was used to compare statistical difference between means. Multivariate logistic regression analysis was performed to determine variables associated with Mets and associated risk. A P value $<0.05$ was considered statistically significant.

\subsection{Ethical Approval}

The study was approved by the Kenyatta National Hospital-University of Nairobi Ethics and Research Committee (Permit No. KNH-ERC/A/232) and the National Commission for Science, Technology and Innovation (NACOSTI) Permit No. NACOSTI/P/16/83452/10118. Study participants gave a written informed consent.

\section{Results}

\subsection{Demographic Characteristics}

Socio demographic characteristics of the respondents are presented in Table 1. Overall, 153 Type 2 Diabetic patients participated in the study. Their mean age was 56.1 years. Over half of them $(58.6 \%)$ were aged between 50-69 years. One 
hundred and nineteen $(119 ; 77.8 \%)$, with a mean age of 56.8 years had poor glycemic control $(\mathrm{HbA1C}>7 \%)$. The highest proportion $(41.2 \%)$ of the study participants were farmers and about one third (31.3\%) were engaged in business. In addition most of the participants had attained primary education $(54.9 \%)$, owned a mobile phone $(96.1 \%)$, owned a house $(75.8 \%)$, were married $(84.3 \%)$ and lived in rural areas $(62.1 \%)$.

Table 1. Socio-demographic characteristics of the participants.

\begin{tabular}{|c|c|c|}
\hline Parameters Category & & Totals n (\%) \\
\hline \multirow{2}{*}{ Gender } & Male & $62(40.5)$ \\
\hline & Female & $91(59.5)$ \\
\hline \multirow{5}{*}{ Age } & $20-49$ & $12(7.8)$ \\
\hline & $40-49$ & $29(19.1)$ \\
\hline & $50-59$ & $46(30.5)$ \\
\hline & $60-69$ & $43(28.1$ \\
\hline & $70-79$ & $23(15.1)$ \\
\hline \multirow{4}{*}{ Marital status } & Single & $16(10.5)$ \\
\hline & Married & $129(84.3)$ \\
\hline & Separated/divorced & $5(3.3)$ \\
\hline & widowed & $3(2.0)$ \\
\hline \multirow{4}{*}{ Highest Education Level } & Primary & $84(54.9)$ \\
\hline & Secondary & $54(35.3)$ \\
\hline & Tertiary & $14(9.2)$ \\
\hline & No formal education & $1(0.7)$ \\
\hline \multirow{5}{*}{ Occupation } & formal employment & $6(3.9)$ \\
\hline & Casual employment & $10(6.5)$ \\
\hline & Farming & $63(41.2)$ \\
\hline & Business & $49(32.0)$ \\
\hline & unemployed & $25(17.1)$ \\
\hline \multirow{2}{*}{ Location of residence } & Rural & $95(62.1)$ \\
\hline & Urban & $58(37.9)$ \\
\hline \multirow{2}{*}{ House ownership } & Own house & $116(75.8)$ \\
\hline & Rental house/others & $37(24.2)$ \\
\hline \multirow{4}{*}{ No of people in $\mathrm{HH}$} & None & $24(15.7)$ \\
\hline & $1-2$ person & $56(36.6)$ \\
\hline & 3-4 person & $48(31.4)$ \\
\hline & 5 person or more & $25(16.3)$ \\
\hline \multirow{3}{*}{ Income } & $<1000$ & $72(47.1)$ \\
\hline & $>1000-10000$ & $55(35.9)$ \\
\hline & $>10000$ & $26(17.0)$ \\
\hline \multirow{5}{*}{ Assets } & Radio & $136(88.9)$ \\
\hline & Television & $120(78.4)$ \\
\hline & Mobile phone & $147(96.1)$ \\
\hline & Bicycle & $37(24.2)$ \\
\hline & Vehicle & $23(15.0)$ \\
\hline
\end{tabular}

$\mathrm{n}$ represents the number of participants while (\%) represents the percentage HH-house hold.

\subsection{Clinical and Lifestyle Characteristic}

As shown in Table 2 , less than half $(46.4 \%)$ of the study participants had a family history of diabetes. Of these participants who had a family history of diabetes, $35.3 \%$ had poor glycemic control. About half (47.1\%) of the participants had a complication as a co-morbidity to Type 2 diabetes. These complications included retinopathy $(23.5 \%)$, arthritis $(11.8 \%)$, lower limb extremity problems $(9.8 \%)$, nephropathy $(1.3 \%)$ and neuropathy (2.6\%). Majority $(96.1 \%)$ of the respondents did not take alcohol. Over half of the participants $(53.6 \%)$ were able to meet the recommended physical activity level as per the WHO guidelines ( $\geq 600 \mathrm{MET} /$ week) with only $5.9 \%$ being vigorously active ( $>3000 \mathrm{MET} /$ week) (Table 2$)$.
Table 2. Clinical and lifestyle characteristics of the participants.

\begin{tabular}{lll}
\hline Parameter & & Total n (\%) \\
\hline \multirow{2}{*}{ FHD } & Yes & $71(46.4)$ \\
& No & $82(53.6)$ \\
& Retinopathy & $36(23.5)$ \\
Complication & Nephropathy & $2(1.3)$ \\
& Neuropathy & $4(2.8)$ \\
& Foot disease & $15(9.8)$ \\
Alcohol intake & Arthritis & $18(11.8)$ \\
& Yes & $6(3.9)$ \\
PAL & No & $147(96.1)$ \\
& light & $71(46.4)$ \\
& moderate & $73(47.7)$ \\
& vigorous & $9(5.9)$ \\
YLWD & 1-4 years & $89(58.2)$ \\
& 5-9 years & $30(19.6)$ \\
& 10-14 years & $19(12.4)$ \\
& 15-19 years & $10(6.5)$ \\
\hline
\end{tabular}

$\mathrm{n}$ represents the number of participants while $(\%)$ represents the percentage. FHD; family history of diabetes, YLWD; years lived with diabetes, PAL; physical activity level.

As shown in Table 3, majority (82.4\%) of the respondents were on oral hypoglycemic agents as a monotherapy; $12.4 \%$ on insulin as a monotherapy and only $5.2 \%$ took a combination of insulin and oral hypoglycemic agents. Most (92.8\%) of the study participants monitored their blood glucose level but did not do other routine examinations recommended for Type 2 diabetes patients, including foot examination $(125 ; 81.7 \%)$, eye examination $(115 ; 75.2 \%)$, lipid profile $(116 ; 75.8 \%)$ and HbAlc $(114 ; 74.5 \%)$. More than half $(64.1 \%)$ of the participants were unable to work as well as before and only $15.0 \%$ had not had a change in lifestyle due to diabetes.

\subsection{Screening, Treatment and Effect of Diabetes on the Patient Life}

Table 3. Screening, treatment and effect of diabetes on the patient life.

\begin{tabular}{lll}
\hline Parameter & & Total n (\%) \\
\hline Screening & & \\
Blood glucose & Yes & $142(92.8)$ \\
monitoring & No & $9(5.9)$ \\
Hba1c & Yes & No $2(14.4)$ \\
& Yes & $131(85.6)$ \\
Lipid profile & No & $19(12.4)$ \\
Eye & Yes & $134(87.6)$ \\
examination & No & $23(15.0)$ \\
Foot & Yes & $130(85.0)$ \\
examination & No & $11(7.2)$ \\
Current & Oral medication & $142(92.8)$ \\
treatment & Oral medication and insulin injection & $126(82.4)$ \\
& Insulin injection & $19(12.2)$ \\
How the & Unable to work as well as before & $98(64.1)$ \\
disease has & Unable to work completely & $10(6.5)$ \\
affected life & Family life & $20(13.1)$ \\
& Socially & $2(1.3)$ \\
\hline
\end{tabular}

$\mathrm{n}$ represents the number of participants while $(\%)$ represents the percentage. Metabolic syndrome (MetS) and associated Mets risk factors.

Prevalence of MetS, MetS and cardiovascular risk factors 
as well as antropometry and biochemical parameters are as the prevalence of Mets was $86.3 \%$ with no significant shown in Table 4, Table 5 and Table 6. As shown in Table 4, difference among male and female patient.

Table 4. Prevalence of MetS based on WHO criteria.

\begin{tabular}{|c|c|c|c|c|}
\hline \multirow{2}{*}{ Metabolic syndrome status } & \multicolumn{3}{|l|}{ Gender } & \multirow{2}{*}{$\chi^{2}$ (P values) } \\
\hline & Male n (\%) & Female n (\%) & Total n (\%) & \\
\hline Yes & $54(35.3)$ & $78(51.0)$ & $132(86.3)$ & $2.658(0.103)$ \\
\hline No & $8(5.2)$ & $13(8.5)$ & $21(13.7)$ & \\
\hline
\end{tabular}

$\mathrm{n}$ represents the number of participants while $(\%)$ represents the percentage.

Chi-square $\left(\chi^{2}\right)$ test; statistical significance at $\mathrm{p}$ value $<0.05$.

As shown in Table 5 majority of the participants; $90.8 \%$ and $86.9 \%$ had high $\mathrm{WC}$ and WHR. The prevalence of hypertension was seen in $65.7 \%$ of the participants and raised TG in $64.7 \%$. About a third of the participants $(28.8 \%)$ had reduced serum HDL cholesterol levels with the prevalence being statistically significant higher $(\mathrm{p}=0.000)$ among women than men. Statistically significant differences $(p=0.0$ and $\mathrm{p}<0.01$ ) were also observed among gender in prevalence's of those participants who were obese and those who had increased WC.
As shown in Table 6, there was no significant difference in the mean body mass index (BMI) of the participants However, there was a statistically significant difference in the WHR ( $\mathrm{P}=0.001$ and $\mathrm{WC}(\mathrm{P}=0.032)$ among males and females participants with males having a higher mean for both measurements. There was also significant difference $(p=$ 0.045 ) between the mean TG among gender with females having a higher level $(2.39 \mathrm{mmol} / \mathrm{l})$ as compared to males $(2.03 \mathrm{mmol} / \mathrm{l})$. There was no significant difference in all the other parameters assayed (Table 6).

Table 5. Prevalence of the various components of metabolic syndrome components and other cardiovascular disease risk factors among the study population stratified by Gender.

\begin{tabular}{|c|c|c|c|c|}
\hline Parameter & Total n (\%) & Male n (\%) & Female n $(\%)$ & $\chi 2$ (P values) \\
\hline $\mathrm{BMI}>30 \mathrm{Kg} / \mathrm{M}^{2}$ & $33(21.6)$ & $8(5.2)$ & $25(16.3)$ & $4.627(0.037)^{*}$ \\
\hline High WHR & $133(86.9)$ & $57(37.3)$ & $76(49)$. & $2.300(0.129)$ \\
\hline elevated TG & $99(64.7$ & $39(25.5)$ & $60(39.2)$ & $0.148(0.700)$ \\
\hline Dyslipidemia & $106(69.3)$ & $41(26.8)$ & $65(42.5)$ & $0.487(0.485)$ \\
\hline Elevated BP ${ }^{\text {a }}$ & $100(65.7)$ & $41(26.8)$ & $59(38.6)$ & $0.027(0.869)$ \\
\hline \multicolumn{5}{|l|}{ Cardiovascular risk factors } \\
\hline Reduced serum HDL-c ${ }^{b}$ & $44(29.7)$ & $6(3.9)$ & $38(24.8)$ & $18.524(0.000) *$ \\
\hline Elevated $\mathrm{BP}^{\mathrm{b}}$ & $111(72.5)$ & $47(30.7)$ & $64(41.8)$ & $0.555(0.0456) *$ \\
\hline Elevated LDL-c & $68(44.4)$ & $27(17.6)$ & $41(26.8)$ & $0.034(0.854)$ \\
\hline Elevated TC & $64(41.8)$ & $24(15.7)$ & $40(26.1)$ & $0.000(0.983)$ \\
\hline
\end{tabular}

$\mathrm{n}$ represents the number of participants while $(\%)$ represents the percentage chi-square $(\chi 2)$ test; $*$ statistical significance at $\mathrm{p}$ value $<0.05 \mathrm{BMI}$ obese $>30 \mathrm{~kg} / \mathrm{m}^{2}$, Elevated Waist hip ratio (WHR) $>0.9$ for men and $>1.0$ for women, Elevated blood pressure ${ }^{\text {a }}>140 / 90 \mathrm{mmHg}$ or treatment of previously diagnosed hypertension (WHO criteria); Elevated blood pressure ${ }^{b}>130 / 80 \mathrm{mmHg}$ or treatment of previously diagnosed hypertension (ACC criteria), Reduced serum HDL cholesterol (a) $<0.9 \mathrm{mmol} / \mathrm{L}$ for men or $<1.0 \mathrm{mmol} / \mathrm{L}$ for women or specific treatment for this abnormality (WHO criteria); Reduced serum HDL cholesterol $^{\mathrm{b}}<1.0 \mathrm{mmol} / \mathrm{L}$ for men or $<1.3 \mathrm{mmol} / \mathrm{L}$ for women or specific treatment for this abnormality (ADA criteria), Elevated triglycerides (TAG) $>1.7$ $\mathrm{mmol} / \mathrm{L}$ or specific treatment for this abnormality (both criteria), Waist circumference $(\mathrm{WC}) \geq 94 \mathrm{~cm}$ for men or $\geq 80 \mathrm{~cm}$ for women, Elevated TC $>5.2 \mathrm{mmol} / 1$, Elevated LDL-cholesterol $>2.6 \mathrm{mmol} / 1$.

Table 6. Anthropometric, clinical and biochemical parameters of patients with Type 2 Diabetes stratified by gender and MetS.

\begin{tabular}{|c|c|c|c|c|c|c|c|}
\hline \multirow{2}{*}{ Parameter } & \multirow{2}{*}{ Total } & \multicolumn{2}{|l|}{ Gender } & \multirow{2}{*}{ P values } & \multicolumn{2}{|c|}{ Presence of MetS } & \multirow{2}{*}{$\begin{array}{l}\text { p value } \\
(<0.05)\end{array}$} \\
\hline & & Male & Female & & Yes & No & \\
\hline $\mathrm{BMI}\left(\mathrm{Kg} / \mathrm{M}^{2}\right)$ & $27.03 \pm 4.70$ & $26.88 \pm 4.11$ & $27.13 \pm 5.08$ & 0.750 & $27.21 \pm 4.77$ & $25.66 \pm 3.98$ & 0.188 \\
\hline $\mathrm{WC}(\mathrm{cm})$ & $100.84 \pm 9.58$ & $102.85 \pm 9.03$ & $99.47 \pm 9.76$ & $0.032 *$ & $101.70 \pm 9.23$ & $94.44 \pm 10.02$ & $0.002 *$ \\
\hline $\mathrm{HC}(\mathrm{cm})$ & $105.0 \pm 9.68$ & $103.63 \pm 8.89$ & $105.93 \pm 10.12$ & 0.149 & $105.95 \pm 9.33$ & $97.89 \pm 9.60$ & $0.001 *$ \\
\hline WHR & $0.96 \pm 0.09$ & $1.00 \pm 0.078$ & $0.94 \pm 0.098$ & $0.001^{*}$ & $0.96 \pm 0.097$ & $0.97 \pm 0.069$ & 0.735 \\
\hline $\mathrm{TC}(\mathrm{mmol} / \mathrm{L})$ & $4.97 \pm 1.1 .22$ & $4.80 \pm 1.22$ & $5.09 \pm 1.231$ & 0.171 & $5.00 \pm 1.26$ & $4.81 \pm 0.94$ & 0.540 \\
\hline $\mathrm{TG}(\mathrm{mmol} / \mathrm{L})$ & $2.24 \pm 1.09$ & $2.02 \pm 0.92$ & $2.38 \pm 1.17$ & $0.045^{*}$ & $2.35 \pm 1.09$ & $1.41 \pm 1.0 .62$ & $0.001^{*}$ \\
\hline HDL (mmol/L) & $1.39 \pm 0.37$ & $1.38 \pm 0.35$ & $1.38 \pm 0.38$ & 0.920 & $1.36 \pm 0.35$ & $1.59 \pm 0.41$ & $0.011^{*}$ \\
\hline $\mathrm{LDL}(\mathrm{mmol} / \mathrm{L})$ & $2.57 \pm 1.07$ & $2.52 \pm 1.12$ & $2.61 \pm 1.04$ & 0.574 & $2.57 \pm 1.08$ & $2.57 \pm 0.99$ & 0.917 \\
\hline HbAlc (\%) & $8.48 \pm 1.86$ & $8.65 \pm 1.99$ & $8.37 \pm 1.76$ & 0.375 & $8.48 \pm 1.89$ & $8.52 \pm 1.64$ & 0.994 \\
\hline $\mathrm{FBG}(\mathrm{mmol} / \mathrm{L})$ & $11.01 \pm 3.39$ & $11.28 \pm 3.67$ & $10.83 \pm 3.19$ & 0.422 & $10.91 \pm 3.09$ & $11.75 \pm 5.17$ & 0.328 \\
\hline $\mathrm{DP}(\mathrm{mmHg})$ & $88.90 \pm 9.55$ & $88.69 \pm 8.56$ & $89.03 \pm 10.21$ & 0.830 & $89.66 \pm 9.78$ & $83.17 \pm 4.77$ & $0.006^{*}$ \\
\hline $\mathrm{SP}(\mathrm{mmHg})$ & $143.78 \pm 20.09$ & $142.77 \pm 20.35$ & $144.47 \pm 1999$ & 0.609 & $145.80 \pm 20.16$ & $128.67 \pm 11.47$ & $0.001 *$ \\
\hline
\end{tabular}

*statistical significance at $\mathrm{p}<0.05$; (a) independent $\mathrm{t}$ test

Data are presented as mean \pm standard deviation of the mean. BMI: body mass index, HC: hip circumference, WHR: waist-to-hip ratio, SP: systolic blood pressure, DP: diastolic blood pressure, LDL low density lipoprotein, TC: total cholesterol and HbAlc-glycated hymoglobin 


\subsection{Association of Patient Characteristics with MetS and Associated Risks}

The association of patient characteristics with metabolic syndrome indicators and associated risk is as shown in Table 7. As shown in Table 7 occupation status was associated with increased WHR with reduced risk seen in patient with formal employment, farming and business $(\mathrm{OR}=0.017, \mathrm{p}=0.012$; $\mathrm{OR}, 0.037, \mathrm{P}=0.028 \& \mathrm{OR}=0.07, \mathrm{P}<0.01$ ) respectively. Additionally participant who had a family history of diabetes $(\mathrm{OR}=6.391, \mathrm{P}<0.01)$ and those drinking alcohol $(\mathrm{OR}=32.64$, $\mathrm{P}=0.011$ ) were significantly associated with obesity (Table 7). Compared to patient earning an income of Ksh. 500-999 per month, participants earning an income of Ksh. 50009,999 and $>10,000$ were significantly associated with elevated DBP $(\mathrm{OR}=5.648, \mathrm{P}=0.046 \& \mathrm{OR}=5.326, \mathrm{P}=0.042)$ with reduced risk as income increases (Table 7). Moreover partcipants earning an income of $>10000$ were associated with reduced risk of MetS $(\mathrm{OR}=0.037, \mathrm{P}=0.018)$ (Table 7). In addition as shown in Table 7, patient who had secondary education were significantly associated with elevated TG $(\mathrm{OR}=3.807, \mathrm{P}=0.008)$ and elevated $\mathrm{BP}(\mathrm{OR}=0.323, \mathrm{P}=0.031)$ compared to those who had only attained primary education. Patient who had lived with diabetes for $\geq 15$ years were also significantly associated with elevated $\mathrm{TG}(\mathrm{OR}=29.308$, $\mathrm{P}=0.014$ ) (Table 7). Gender, Marital status and type residence showed some association with elevated HDL. Compared to male, female were significantly associated with reduced HDL $(\mathrm{OR}=0.047, \mathrm{P}=0.000)$ (Table 7). Also as shown in Table 7 patient who were living in the urban area were significantly associated with reduced compared to the single and those living in the rural areas $\mathrm{HDL}(\mathrm{OR}=0.065, \mathrm{P}<0.01 \mathrm{OR}=$ $0.207, \mathrm{p}=0.037$ ) respectively. Additionally as shown in Table 7 patient who lived in the urban areas were associated with elevated $\mathrm{BP}(\mathrm{OR}=0.276, \mathrm{p}=0.033)$. Other characteristic of the participants showed some association with Mets Component and cardiovascular risk that were not statistically significant.

Table 7. Associations of patient Characteristics and Metabolic syndrome components.

\begin{tabular}{|c|c|c|c|c|c|c|}
\hline \multirow{2}{*}{ Parameter } & & \multicolumn{5}{|l|}{ High WHR } \\
\hline & & n (\%) & OR & $95 \% \mathrm{CI}$ & & P value \\
\hline \multirow{2}{*}{ Gender } & Male & $57(42.9)$ & Ref & & & \\
\hline & Female & $76(57.1)$ & 2.534 & 0.494 & 13.002 & 0.265 \\
\hline \multirow{5}{*}{ Age } & $20-39$ & $11(8.3)$ & Ref & & & \\
\hline & $40-49$ & $22(16.5)$ & 2.833 & 0.071 & 113.376 & 0.580 \\
\hline & $50-59$ & $40(30.1)$ & 2.055 & 0.057 & 74.329 & 0.694 \\
\hline & $60-69$ & $39(29.3)$ & 1.554 & 0.036 & 67.061 & 0.819 \\
\hline & $70-79$ & $21(15.8)$ & 1.505 & 0.023 & 98.465 & 0.848 \\
\hline \multirow{3}{*}{ Marital status } & Single & $12(9.0)$ & Ref & & & \\
\hline & Married & $114(85.7)$ & 1.380 & 0.119 & 15.967 & 0.796 \\
\hline & others & $7(5.3)$ & 2.512 & 0.050 & 125.342 & 0.644 \\
\hline \multirow{3}{*}{ Education } & Primary & $72(54.1)$ & Ref & & & \\
\hline & Secondary & $49(36.8)$ & 0.230 & 0.041 & 1.289 & 0.095 \\
\hline & Tertiary & $12(9)$ & 0.396 & 0.035 & 4.522 & 0.456 \\
\hline \multirow{5}{*}{ Occupation } & unemployed & $25(18.8)$ & Ref & & & \\
\hline & Formal employment & $2(1.5)$ & 0.043 & 0.001 & 1.633 & 0.090 \\
\hline & Casual employment & $8(6.0)$ & 0.017 & 0.001 & 0.403 & $0.012 *$ \\
\hline & Farming & $57(42.9)$ & 0.037 & 0.002 & 0.704 & $0.028 *$ \\
\hline & Business & $41(30.8)$ & 0.007 & 0.000 & 0.283 & $0.009 *$ \\
\hline \multirow{2}{*}{ Type of residence } & Rural & $85(63.9)$ & Ref & & & \\
\hline & Urban & $48(36.1)$ & 3.382 & 0.559 & 20.455 & 0.185 \\
\hline \multirow{2}{*}{ house ownership } & Own house & $100(75.2)$ & Ref & & & \\
\hline & Rental house \&others & $33(24.9)$ & 0.196 & 0.029 & 1.312 & 0.093 \\
\hline \multirow{4}{*}{ No. of HH members } & None & $19(14.3)$ & Ref & & & \\
\hline & $1-2$ person & $50(37.6)$ & 0.476 & 0.056 & 4.048 & 0.497 \\
\hline & 3-4 person & $43(32.3)$ & 0.533 & 0.079 & 3.578 & 0.517 \\
\hline & 5 person or more & $21(15.8)$ & 0.766 & 0.107 & 5.504 & 0.791 \\
\hline \multirow{4}{*}{ Income (Ksh) } & $500-1000$ & $65(48.9)$ & Ref & & & \\
\hline & $>1000-4999$ & $27(20.3)$ & 1.796 & 0.350 & 9.214 & 0.483 \\
\hline & $>5000-9999$ & $19(14.3)$ & 1.292 & 0.120 & 13.903 & 0.832 \\
\hline & $\geq 10000$ & $22(16.5)$ & 0.781 & 0.077 & 7.966 & 0.835 \\
\hline \multirow{2}{*}{ FHD } & No & $63(47.4)$ & Ref & & & \\
\hline & Yes & $70(52.6)$ & 2.572 & 0.596 & 11.093 & 0.205 \\
\hline \multirow{2}{*}{ Complication } & Yes & $64(48.1)$ & Ref & & & \\
\hline & No & $69(51.9)$ & 2.350 & 0.552 & 10.012 & 0.248 \\
\hline \multirow{5}{*}{ YLWD } & $1-4.99$ years & 69 (51.9) & Ref & & & \\
\hline & $>5-9.99$ years & $24(18.0)$ & 3.230 & 0.562 & 18.570 & 0.189 \\
\hline & $>10-14.99$ years & $24(19.0)$ & 4.463 & 0.651 & 30.604 & 0.128 \\
\hline & $>15-19.99$ years & $16(12.0)$ & 3.322 & 0.182 & 60.572 & 0.418 \\
\hline & $\geq 20$ years & & 0.000 & 0.000 & . & 0.999 \\
\hline \multirow{2}{*}{ Alcohol intake } & No & $127(95.5)$ & Ref & & & \\
\hline & Yes & $6(4.6)$ & 0.000 & 0.000 & & 0.999 \\
\hline
\end{tabular}




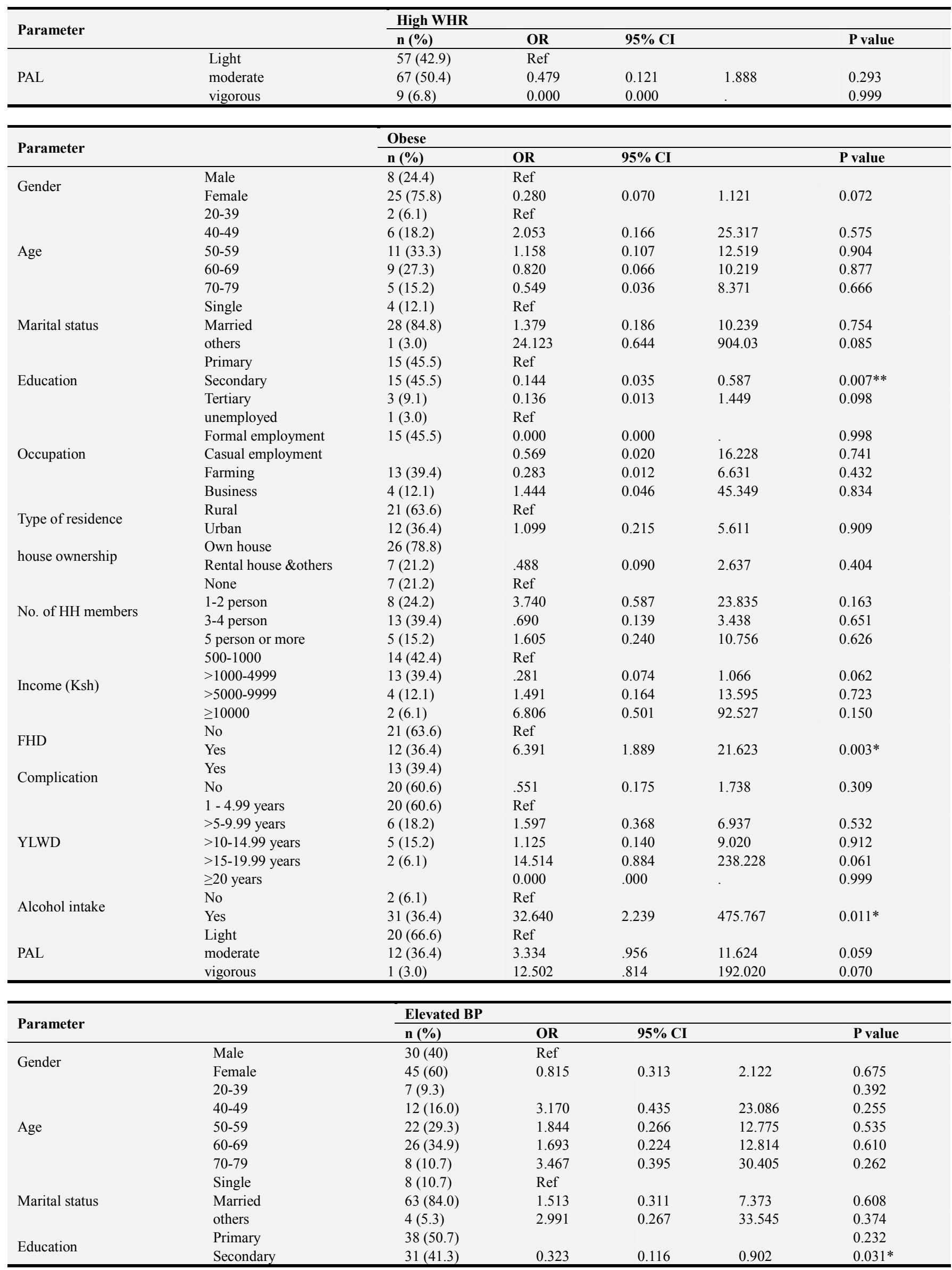




\begin{tabular}{|c|c|c|c|c|c|c|}
\hline \multirow{3}{*}{ Parameter } & & \multicolumn{5}{|c|}{ Elevated BP } \\
\hline & & \multirow{2}{*}{$\begin{array}{l}\mathbf{n}(\mathbf{\%}) \\
6(8.0)\end{array}$} & \multirow{2}{*}{$\begin{array}{l}\text { OR } \\
0.513\end{array}$} & \multicolumn{2}{|c|}{$95 \%$ CI } & \multirow{2}{*}{$\begin{array}{l}\text { P value } \\
0.467\end{array}$} \\
\hline & \multirow{2}{*}{$\begin{array}{l}\text { Tertiary } \\
\text { unemployed }\end{array}$} & & & 0.085 & 3.103 & \\
\hline \multirow{5}{*}{ Occupation } & & $2(2.6)$ & Ref & & & \\
\hline & Formal employment & $5(6.4)$ & 0.067 & 0.002 & 1.947 & 0.116 \\
\hline & Casual employment & $29(37.2)$ & 0.739 & 0.102 & 5.336 & 0.765 \\
\hline & Farming & $25(32.1)$ & 0.515 & 0.138 & 1.918 & 0.323 \\
\hline & Business & $17(21.8)$ & 0.444 & 0.113 & 1.741 & 0.244 \\
\hline \multirow{2}{*}{ Type of residence } & Rural & $52(69.3)$ & Ref & & & \\
\hline & Urban & $23(30.7)$ & 0.276 & 0.085 & 0.898 & $0.033^{*}$ \\
\hline \multirow{3}{*}{ house ownership } & Own house & $61(81.3)$ & Ref & & & \\
\hline & Rental house \&others & $14(18.7)$ & 0.719 & 0.214 & 2.416 & 0.594 \\
\hline & None & $11(14.7)$ & Ref & & & \\
\hline \multirow{3}{*}{ No. of HH members } & 1-2 person & $29(38.7)$ & 1.953 & 0.426 & 8.957 & 0.389 \\
\hline & $3-4$ person & $25(33.3)$ & 1.721 & 0.460 & 6.441 & 0.420 \\
\hline & 5 person or more & $10(13.3)$ & 1.186 & 0.319 & 4.415 & 0.799 \\
\hline \multirow{4}{*}{ Income (Ksh) } & $500-1000$ & $36(48.0$ & Ref & & & \\
\hline & $>1000-4999$ & $12(16.0)$ & 2.077 & 0.395 & 10.916 & 0.388 \\
\hline & $>5000-9999$ & $9(12.0)$ & 5.648 & 1.030 & 30.979 & $0.046^{*}$ \\
\hline & $\geq 10000$ & $18(24.0)$ & 5.326 & 1.065 & 26.628 & $0.042 *$ \\
\hline \multirow{2}{*}{ FHD } & No & $36(48.0)$ & Ref & & & \\
\hline & Yes & $39(52.0)$ & 1.483 & 0.609 & 3.610 & 0.386 \\
\hline \multirow{3}{*}{ Complication } & Yes & $36(48.0)$ & & & & \\
\hline & No & $39(52.0)$ & 0.560 & 0.238 & 1.315 & 0.183 \\
\hline & $1-4.99$ years & $33(44.0)$ & Ref & & & \\
\hline \multirow{4}{*}{ YLWD } & $>5-9.99$ years & $16(21.3)$ & 2.085 & 0.163 & 26.690 & 0.572 \\
\hline & $>10-14.99$ years & $14(18.7)$ & 2.484 & 0.171 & 36.111 & 0.505 \\
\hline & $>15-19.99$ years & $12(16.0)$ & 0.428 & 0.020 & 9.143 & 0.587 \\
\hline & $\geq 20$ years & & 2.184 & 0.119 & 40.040 & 0.599 \\
\hline \multirow{3}{*}{ Alcohol intake } & No & $4(5.3)$ & Ref & & & \\
\hline & Yes & $71(94.7)$ & 1.012 & 0.137 & 7.493 & 0.991 \\
\hline & Light & $33(44.0)$ & Ref & & & \\
\hline \multirow[t]{2}{*}{ PAL } & moderate & $37(49.3)$ & .957 & 0.840 & 0.349 & 2.021 \\
\hline & vigorous & $5(6.7)$ & 1.027 & 0.631 & 0.081 & 4.907 \\
\hline
\end{tabular}

Ref-reference point.

$\mathrm{n}$ represents the number of participants while (\%) represents the percentage.

OR - Odds ratio; $95 \%$ CI- $95 \%$ confidence interval; $* *$ statistical significance at $\mathrm{p}$ value $<0.05$, ref -reference point.

BMI obese $>30 \mathrm{~kg} / \mathrm{m}^{2}$, Elevated Waist hip ratio (WHR) $>0.9$ for men and $>1.0$ for women, High blood pressure $>140 / 90 \mathrm{mmHg}$ or treatment previously diagnosed hypertension.

HH-house hold; FHD; family history of diabetes, YLWD; years lived with diabetes, PAL; physical activity level.

Table 7. Continued.

\begin{tabular}{|c|c|c|c|c|c|c|}
\hline \multirow{2}{*}{ Parameters } & & \multicolumn{5}{|c|}{ Elevated HDL } \\
\hline & & \multirow{2}{*}{$\begin{array}{l}\mathbf{n ~ ( \% )} \\
4(9.1)\end{array}$} & \multirow{2}{*}{$\begin{array}{l}\text { OR } \\
\text { Ref }\end{array}$} & \multicolumn{2}{|l|}{$95 \%$ CI } & \multirow[t]{2}{*}{ Pvalve } \\
\hline Gender & Male & & & & & \\
\hline & Female & $40(90.9)$ & 0.047 & 0.011 & .210 & $0.000 * *$ \\
\hline \multirow[t]{5}{*}{ Age } & $20-39$ & $5(11.4)$ & & & & 0.656 \\
\hline & $40-49$ & $11(25)$ & 1.457 & 0.170 & 12.513 & 0.732 \\
\hline & $50-59$ & $14(31.8)$ & 2.542 & 0.291 & 22.168 & 0.398 \\
\hline & $60-69$ & $9(20.5)$ & 4.270 & 0.443 & 41.143 & 0.209 \\
\hline & $70-79$ & $5(11.4)$ & 3.494 & 0.247 & 49.417 & 0.355 \\
\hline \multirow[t]{3}{*}{ Marital status } & Single & $5(11.4)$ & Ref & & & \\
\hline & Married & $38(86.4)$ & 0.065 & 0.010 & .422 & $0.004 * *$ \\
\hline & Separated /divorced/widowed & $1(23)$ & 0.373 & 0.017 & 8.155 & 0.531 \\
\hline \multirow[t]{3}{*}{ Education } & Primary & $25(56.8)$ & Ref & & & \\
\hline & Secondary & $16(36.4)$ & 0.933 & 0.293 & 2.976 & 0.907 \\
\hline & Tertiary & $3(6.8)$ & 1.755 & 0.171 & 18.012 & 0.636 \\
\hline \multirow[t]{5}{*}{ Occupation } & unemployed & $4(9.1)$ & & & & 0.379 \\
\hline & Formal employment & $3(6.8)$ & 0.903 & 0.074 & 11.018 & 0.936 \\
\hline & Casual employment & $18(40.9)$ & 3.062 & 0.303 & 30.911 & 0.343 \\
\hline & Farming & $10(22.7)$ & 1.731 & 0.320 & 9.374 & 0.524 \\
\hline & Business & $9(20.5)$ & 4.945 & 0.825 & 29.627 & 0.080 \\
\hline \multirow[t]{2}{*}{ Type of residence } & Rural & $25(56.8)$ & Ref & & & \\
\hline & Urban & $19(43.2)$ & 0.207 & 0.047 & .907 & $0.037^{*}$ \\
\hline \multirow[t]{3}{*}{ House ownership } & Own house & $33(75.0)$ & & & & \\
\hline & Rental house and others & $11(25.0)$ & 1.465 & 0.365 & 5.872 & 0.590 \\
\hline & None & $7(15.9)$ & Ref & & & 0.160 \\
\hline
\end{tabular}




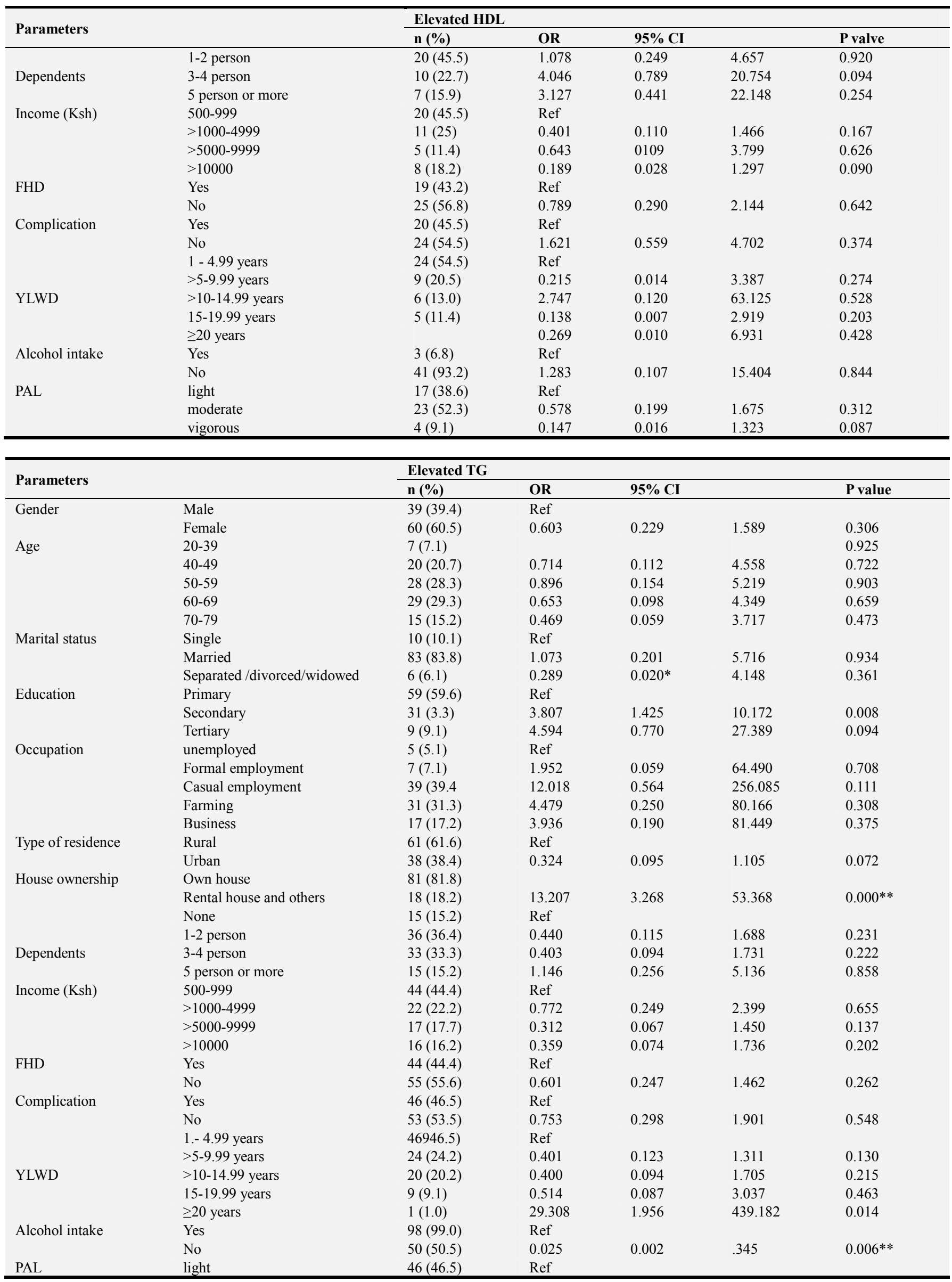




\begin{tabular}{|c|c|c|c|c|c|c|}
\hline \multirow{4}{*}{ Parameters } & & \multicolumn{5}{|c|}{ Elevated TG } \\
\hline & & \multirow{3}{*}{$\begin{array}{l}\mathbf{n}(\%) \\
3(3.0)\end{array}$} & \multirow{2}{*}{$\begin{array}{l}\text { OR } \\
1.483\end{array}$} & \multicolumn{2}{|l|}{$95 \%$ CI } & \multirow{2}{*}{$\begin{array}{l}\text { P value } \\
0.393\end{array}$} \\
\hline & moderate & & & 0.600 & 3.663 & \\
\hline & vigorous & & 2.514 & 0.409 & 15.469 & 0.320 \\
\hline \multirow{2}{*}{ Parameters } & & \multicolumn{4}{|l|}{ MetS } & \\
\hline & & n (\%) & OR & $95 \% \mathrm{CI}$ & & Pvalue \\
\hline \multirow[t]{2}{*}{ Gender } & Male & $54(40.9)$ & Ref & & & \\
\hline & Female & $78(59.1)$ & 0.716 & 0.190 & 2.692 & 0.621 \\
\hline \multirow[t]{5}{*}{ Age } & $20-39$ & $11(8.3)$ & Ref & & & \\
\hline & $40-49$ & $23(17.4)$ & 1.095 & 0.053 & 22.733 & 0.953 \\
\hline & $50-59$ & $40(30.3)$ & 0.730 & 0.038 & 13.969 & 0.835 \\
\hline & $60-69$ & $38(28.8)$ & 0.555 & 0.025 & 12.469 & 0.710 \\
\hline & $70-79$ & $20(15.2)$ & 0.823 & 0.030 & 22.587 & 0.908 \\
\hline \multirow[t]{3}{*}{ Marital status } & Single & $13(9.8)$ & Ref & & & \\
\hline & Married & $113(85.6)$ & 0.990 & 0.142 & 6.908 & 0.992 \\
\hline & Separated /divorced/widowed & $6(3.9)$ & 1.713 & 0.094 & 31.158 & 0.716 \\
\hline \multirow[t]{3}{*}{ Education } & Primary & $73(55.3)$ & Ref & & & \\
\hline & Secondary & $48(36.4)$ & 0.696 & 0.173 & 2.809 & 0.611 \\
\hline & Tertiary & $11(8.3)$ & 4.175 & 0.514 & 33.886 & 0.181 \\
\hline \multirow[t]{5}{*}{ Occupation } & unemployed & $21(15.9)$ & Ref & & & \\
\hline & Formal employment & $4(3.0)$ & 0.318 & 0.015 & 6.885 & 0.465 \\
\hline & Casual employment & $7(5.3)$ & 0.080 & 0.004 & 1.512 & 0.092 \\
\hline & Farming & $57(43.2)$ & 0.102 & 0.007 & 1.542 & 0.100 \\
\hline & Business & $43(32.6)$ & 0.090 & 0.005 & 1.762 & 0.113 \\
\hline \multirow[t]{2}{*}{ Type of residence } & Rural & $86(65.2)$ & Ref & & & \\
\hline & Urban & $46(34.8)$ & 1.829 & 0.368 & 9.095 & 0.460 \\
\hline \multirow[t]{4}{*}{ House ownership } & Own house & $102(87.3)$ & Ref & & & \\
\hline & Rental house and others & $30(22.7)$ & 1.428 & 0.287 & 7.100 & 0.663 \\
\hline & None & $18(13.6)$ & & & & \\
\hline & $1-2$ person & $49(37.2)$ & 0.418 & 0.072 & 2.421 & 0.330 \\
\hline \multirow[t]{2}{*}{ Dependents } & 3-4 person & $44(33.3)$ & 0.140 & 0.019 & 1.050 & 0.056 \\
\hline & 5 person or more & $21(15.9)$ & 0.383 & 0.060 & 2.457 & 0.311 \\
\hline \multirow[t]{4}{*}{ Income (Ksh) } & $500-999$ & $60(45.5)$ & Ref & & & \\
\hline & $>1000-4999$ & $29(45.5)$ & 0.430 & 0.077 & 2.399 & 0.336 \\
\hline & $>5000-9999$ & $18(13.6)$ & 0.357 & 0.047 & 2.714 & 0.319 \\
\hline & $>10000$ & $25(18.9)$ & 0.037 & 0.002 & .572 & 0.018 \\
\hline \multirow[t]{2}{*}{ FHD } & Yes & $61(46.2)$ & Ref & & & \\
\hline & No & $71(53.8)$ & 2.038 & 0.306 & 13.587 & 0.462 \\
\hline \multirow[t]{4}{*}{ Complication } & Yes & $60(47.0)$ & & & & \\
\hline & No & $72(53.0)$ & 0.754 & 0.212 & 2.675 & 0.662 \\
\hline & $1-4.99$ years & $78(59.1)$ & Ref & & & \\
\hline & $>5-9.99$ years & $25(18.9)$ & 1.368 & 0.293 & 6.398 & 0.690 \\
\hline \multirow[t]{3}{*}{ YLWD } & $>10-14.99$ years & $16(12.1)$ & 0.965 & 0.141 & 6.615 & 0.971 \\
\hline & $15-19.99$ years & $9(9.0)$ & 0.952 & 0.075 & 12.056 & 0.970 \\
\hline & $\geq 20$ years & $4(4.0)$ & 7.234 & 0.320 & 163.386 & 0.213 \\
\hline \multirow[t]{2}{*}{ Alcohol intake } & Yes & $4(3.0)$ & Ref & & & \\
\hline & No & $12(97.0)$ & 9.871 & 0.904 & 107.718 & 0.060 \\
\hline PAL & light & $58(43.9)$ & Ref & & & \\
\hline & moderate & $66(50.0)$ & 0.408 & 0.102 & 1.624 & 0.203 \\
\hline & vigorous & $2(6.1)$ & 0.226 & 0.007 & 7.002 & 0.396 \\
\hline
\end{tabular}

Ref: refrence point; $\mathrm{n}$ represents the number of participants while $(\%)$ represents the percentage.

OR - Odds ratio; $95 \%$ CI- 95\% confidence interval; * statistical significance at $\mathrm{p}$ value $<0.05, * *$ statistical significance at $\mathrm{p}$ value $<0.01$ ref -reference point. Reduced HDL cholesterol $<1.0 \mathrm{mmol} / \mathrm{L}$ for men or $<1.3 \mathrm{mmol} / \mathrm{L}$ for women or specific treatment for this abnormality (Elevated triglycerides (TAG) $>1.7$ $\mathrm{mmol} / \mathrm{L}$ or specific treatment for this abnormality.

FHD; family history of diabetes, YLWD; years lived with diabetes, PAL; physical activity level MetS defined as per WHO criteria.

\section{Discussion}

Type 2 Diabetes, metabolic syndrome (MetS) and cardiovascular disorder (CVD) are prevalent chronic conditions of global importance that can be controlled with proper management. $[4,6,8]$ This could result to potential benefit geared toward the patient, the health care system as well economic development $[4,15,41]$. The metabolic syndrome (MetS), a cluster of risk factors which include raised blood pressure, dyslipidemia (raised triglycerides and lowered high-density lipoprotein cholesterol), raised fasting glucose, and central obesity (increased waist circumference) has been shown to increase the risk to type 2 Diabetes by 5 fold and cardiovascular disease by 2 folds [13, 15]. Our current study explored the association between patient 
characteristic and MetS and associated cardiovascular risk. Exploring these association might aid in development of preventive measure therefore improving the quality of life of the diabetes patients.

Overall 153 (59.5 female and 40.5 male) Type 2 Diabetic patient were incorporated into the study. Majority were aged between 50-59 years with an average age of 56.08 years and 56.51 years for those with MetS and poor glycemic control respectively. Indeed, age has been shown to be a risk factor in Type 2 diabetes, MetS and associated cardiovascular risk [19] Studies conducted on Type 2 Diabetes patients have reported a high prevalence of Type 2 diabetes and MetS in older people ( $>50$ years) and this is in congruent with our current study [12, 17, 18, 42].

Prevalence study on MetS, MetS risk and associated CVD risk factors in Type 2 diabetes patients have revealed different rates in different places, depending on definition criteria used [11, 14, 43]. Our study reported a high prevalence $(>80 \%)$ of MetS using the WHO criteria which was comparable among gender. These results are agreement to previous studies which reported high prevalence's ( $>70 \%)$ of MetS in Type 2 Diabetes patients supported by [21, 44] Presence of MetS in Type 2 Diabetes patient increases the risk of microvascular macrovascular complications in addition to cardiovascular disorders [35, 36]. The high prevalence of Mets in the current study might have been due increased risk factors in the study participant. In fact, most (88.9\%) of the Type 2 diabetes patients who participated in this study had three or more MetS component risk factors and this, might explain the high prevalence of Mets. Similar findings were reported by Raman et al [45] and Ogbera 2010 [18] and are in support of the current study. Increased WC was the most prevalent component, followed by high WHR, elevated serum TG and elevated blood pressure; with most of the participants recording higher figures than the cut-off point (Table 5). Higher overall mean above the agreed cut off points for BMI, WC, TG, SBP, FBG, and HbAlc were noted in the current study. The male participants had statistically significantly higher mean WC, WHR and female higher statistically significantly TG. The current study is in agreement with other studies that have shown an association of dyslipidemia with obesity characterized by BMI $>30 \mathrm{~kg} / \mathrm{m}^{2}$, elevated WC, high WHR, poor glycemic control and elevated BP. [46-49]. This association is a key risk factor to MetS, CVD as well as progression of Type 2 diabetes complication Over half of the patient had elevated TC and LDL-c key indicator of cardiovascular risk factor related to progression of MetS, Type 2 Diabetes and CVD [4, 6, 8]. Moreover combination of these risk factors complicates the management thus escalating the problem further $[4,6,50$ 52].

Majority (77.8\%) of the participants had poor glycemic control, with an average mean HbAlc of $8.49 \%$. Similar findings were reported by Raman et al., and Moreira et al $[45,46]$. Poor glycemic control $(\mathrm{HbA} 1 \mathrm{c}>7 \%)$ poses a major risk to Type 2 diabetes patients and those with MetS. Moreover, combinations of risk factors such as increased
WC, elevated TC, increased BP and reduced HDL, elevated TC, and elevated LDL-c may lead to poor glycemic control, development of cardiovascular, micro vascular and macro vascular complications in Type 2 diabetes $[1,32]$. A strong association of poor glycemic control, hypertension, dyslipidemia and central obesity with MetS as well as Type 2 diabetes has been reported [46-49]. Elevated WC as well as high WHR or BMI and dyslipidemia have been associated with abdominal obesity, which is a major cause of insulin resistance; one of the important risk factors of MetS and type diabetes [6,7]. The condition worsens in the presence of elevated blood pressure, one of the major complications in Type 2 Diabetes and key risk to CVD [6, 7, 50, 51].

Patient characteristics have been associated with increased risk to MetS, associated risk and CVD risk factors. Studying their association with Mets and associated risk is paramount as strategic way for preventive measures Studies have reported varying prevalence of MetS among gender, as well as other patient characteristics. A study by Kengen et al., [17] reported a significant high prevalence of MetS in women compared to men. A study by Kaduka et al, [22] in a general population showed significant association of MetS with age, level of education, monthly income and social economic status with advanced age, wealth quantile and higher education being strongly associated with MetS. A study by Tadewos et al., [53] on Type 2 Diabetes also revealed significant association of gender, occupation, duration of diabetes and nutrition status with MetS.

Moreover our current study showed different association of patient characteristics with MetS risk factors and selected CVD risk factors. (Table 7) using multiple logistic regression. Studies have shown that occupation status of patient have been associated with improved social economic status that usually leads to adaptation of behavior traits that increase the metabolic risk in patient with Type 2 diabetes [55]. Our study was unique as it showed some association of economic status with increased MetS risk with patient having a higher income being associated with elevated blood pressure (BP). Patient occupation status also showed some significant association with high WHR. A study by Ogunsina et al [56] is in support of the current study as it showed that high social economic status for both men and women was associated with increased odds of overweight/ obesity. Patient with secondary education from the current study were associated with increased odds of obesity and elevated TG. However our current study reported reduced risk of overall MetS as income level increased (Table 7) supporting the evidence that high income levels is associated with reduced risk [54].

Additionally patient with a family history of diabetes and were taking alcohol were also associated with obesity. Patient who had lived with diabetes $\geq 15$ years were also associated with elevated TG. Family history of diabetes (FHD), alcohol intake and increased years with diabetes predisposes Type 2 Diabetes patient to metabolic risk like obesity and dyslipidemia as well as associated complication [57] and this is in support of our current study. Moreover 
gender, marital status and type of residence were associated with reduced HDL with female patient, married patient and patient living in urban areas being significantly associated with increased odds of reduced HDL. This might have been due to adapted behaviour by the patients

Our study had some limitations. The above study was conducted in a Hospital setup on Type 2 Diabetes patients visiting the clinic. Thus the result might not represent a true sample of population, given that some diabetes patients may not be attending the clinic. To find out the true prevalence, a community-based study needs to be conducted and comparison with hospital based studies done. However, several studies conducted on Type 2 Diabetes on prevalence of MetS in different countries have been done on hospital set up, hence making our result comparable.

\section{Conclusion}

A high prevalence of MetS in our current study was noted on Type 2 Diabetes patients using the WHO and harmonized criteria. The most prevalent components of the MetS were elevated WC, increased WHR, and elevated TG and elevated BP. The current study showed that participants with some form of occupation had attained secondary education and earned a higher income was associated with increased MetS risk factors. Income was associated with elevated diastolic blood pressure (DBP), secondary education and years lived with diabetes were associated with elevated $\mathrm{TG}$, while occupation showed some association with high WHR. Additionally Gender, marital status and type of residence were associated with reduced HDL while education, family history of diabetes and alcohol intake was associated with obesity. This calls for an urgent action aimed at preventing the progression of the patients to diabetes complications and cardiovascular problems. Increased surveillance on MetS in Type 2 Diabetes patients need to be hastened and preventive measures (like lifestyle and diet intake modification, doing regular moderate to vigorous intensity physical exercises) put in place to prevent the condition from worsening.

\section{Abbreviations}

MetS-metabolic syndrome; HbA1c: glycated hymoglobin; WHO: World Health Organization; WC: waist circumference; HDL-c: High Density Lipoprotein cholesterol; TG: Triglyceride; Total Cholesterol; LDL-C: Low Density Lipoprotein; BMI: Body Mass Index; WHR: Waist Hip Ratio; CVD: Cardiovascular Disease; TL5H: Thika Level 5 Hospital; DCC: Diabetes Care Centre; NEG: Nutrition Education Group; NEP: Nutrition Education Peer to peer support Group; MET: Metabolic Equivalent; GPO/POD: Glycerol Phosphate Oxidase Peroxidase; CHOD/POD: Cholesterol Oxidase Peroxidase, KNHUoN/ERC: Kenyatta National Hospital-University of Nairobi Ethical Research Committee; NACOSTI: National Commission for Science Technology and Innovation; ADDRF: Africa Doctoral Dissertation Research Fellowship;
APHRC: Africa Population and Health Research Center; IDRC: International Development Research Centre; SD: Standard Deviation; SPSS: Statistical Package for Social Sciences.

\section{Competing Interests}

The authors declare that they have no competing interests.

\section{Acknowledgements}

The support of the Laboratory Technologist David Ngaruma for his support in biochemical data analysis is appreciated. The administration and the staff working in the Diabetes Centre Thika Level 5 hospital is highly appreciated. Additionally the participation and cooperation of the patient involved in the study is greatly appreciated.

\section{Availability of Data and Materials}

All the data collection tool and data are in the custody of Thuita Ann and are available on request.

\section{Ethics Approval and Consent to Participate}

Ethical approval was obtained from Kenyatta National Hospital and University of Nairobi Ethical Committee (Permit No. KNH-ERC/A/232) and while administrative approval was obtained from the National Commission for Science, Technology and Innovation (NACOSTI) Permit No. NACOSTI/P/16/83452/10118, the Ministry of Interior and Co-ordination of National Government, County Commissioner Kiambu Permit No. ED.12/1/VOL. IV/92, Ministry of Education Kiambu Permit No. KBU/CDE/HR/4/VOL. II (138) county health officials and health facility administrators. Study participants gave a written informed consent before commencement of data collection.

\section{References}

[1] ADA; American Diabetes Association. Standards of Medical Care in Diabetes - 2018. J Clin Appl Res Educ 2018; 41.

[2] IDF; International DiabetesFederation. Annual report. Belgium: 2014.

[3] IDF; International Diabetes Federation. Annual Report. 2013.

[4] IDF; International Diabetes Federation. IDF Diabetes Atlas, Seventh Edition. Brussesl, Belgium: 2015.

[5] WHO; World Health Organization. Defination, Diagnosis and Classfication of Diabetes Mellitus and its Complication. Geneva, Switzerland: 1998.

[6] WHO; World Health Organization. Global Report on Diabetes. Geneva, Switzerland: 2016. 
[7] IDF; International DiabetesFederation. IDF Diabetes Atlas Eighth edition. 2017. doi: http://dx.doi. org/10.1016/S01406736(16)31679-8.

[8] WHO; World Health Organization. Non-Communicable Progress monitor 2017. Geneva, Switzerland: World Health Organization; 2017.

[9] Siu PM, Yuen QS. Supplementary use of HbAlc as hyperglycemic criterion to detect metabolic syndrome. Diabetol Metab Syndr 2014; 6: 1-7. doi: 10.1186/1758-5996-6-119.

[10] Rhee SY, Park SY, Hwang JK, Son J Il, Chin SO, Kim YS. Metabolic syndrome as an indicator of high cardiovascular risk in patients with diabetes: Analyses based on Korea National Health and Nutrition Examination Survey (KNHANES) 2008. Diabetol Metab Syndr 2014; 6: 1-9.

[11] Osei-yeboah J, Owiredu WKBA, Norgbe GK, Lokpo SY, Gyamfi J, Allotey EA, et al. The Prevalence of Metabolic Syndrome and Its Components among People with Type 2 Diabetes in the Ho Municipality, Ghana: A Cross-Sectional Study. Hindawi Int J Chronic Dis 2017. doi: $10.1155 / 2017 / 8765804$.

[12] Tamang HK, Timilsina U, Thapa S, Singh KP, Shrestha S, Singh P, et al. Prevalence of metabolic syndrome among Nepalese type 2 diabetic patients. Nepal Med Coll J 2013; 15: $50-5$.

[13] Alberti K. G. M. M., Eckel RH, Grundy SM, Zimmet PZ, Cleeman JI, Donato KA, et al. Harmonizing the Metabolic Syndrome; A Joint Interim Statement of the International Diabetes Federation Task Force on Epidemiology and Prevention; National Heart, Lung, and Blood Institute; American Heart Association; World Heart Federation; International. Greenville Avenue, Dallas: 2009. doi: 10.1161/CIRCULATIONAHA.109.192644.

[14] Hajian-Tilaki. k, Heidari. B, Bagherzadeh. M, Hajian-Tilaki. A H. Prevalence of metabolic syndrome and the association with socio-demographic characteristics and physical activity in urban population of Iranian adults: A population study. Diabetes Metab Syndr Clin Res Rev 2014; 8: 170-6. doi: 10.1016/j.dsx.2014.04.012.

[15] Kaur J. A Comprehensive Review on Metabolic Syndrome. Cardiol Res Pract 2014.

[16] Kaimuri M, Mwaniki P, Kombe Y. Prevalence of Metabolic Syndrome and Its Determinants among Women of Reproductive Age In Meru County, Kenya. Kenyan J Nurs Midwifery 2016; 1: 158-70.

[17] Kengne AP, Limen SN, Sobngwi E, Djouogo CFT, Nouedoui C. Metabolic syndrome in type 2 diabetes: comparative prevalence according to two sets of diagnostic criteria in subSaharan Africans. Diabetol Metab Syndr 2012; 4: 1-8. doi: 10.1186/1758-5996-4-22.

[18] Ogbera AO. Prevalence and gender distribution of the metabolic syndrome. Diabetol Metab Syndr 2010; 2: 6-10. doi: 10.1186/1758-5996-2-1.

[19] Nazaimoon W, Mohamud W, Ismail A, Sharifuddin A. Prevalence of metabolic syndrome and its risk factors in adult Malaysians: Results of a nationwide survey. Diabetes Res Clin Pract 2011; 96: 91-7. doi: 10.1016/j.diabres.2011.11.020.

[20] Kaur J. Assessment and Screening of the Risk Factors in Metabolic Syndrome. Med Sci 2014; 2: 140-52. doi: 10.3390/medsci2030140.

[21] Alwan AH, Alhusuny A. Assessment of Metabolic Syndrome and Its Risk Factors among Patients with Type 2 DM at Merjan Teaching Hospital, Al-Hilla City. J Dent Med Sci 2014; 13: 69-75.

[22] Kaduka LU, Kombe Y, Kenya E, Kuria E, Bore JK, Bukania $\mathrm{ZN}$, et al. Prevalence of Metabolic Syndrome Among an Urban Population in Kenya. Diabetes Care 2012; 35: 887-93. doi: $10.2337 / \mathrm{dc} 11-0537$.

[23] Armitage P, Berry G, Matthews JN. Statistical Methods in Medical Research. Wileys Publication; 2008. doi: 1002/978040773666.

[24] Lwanga S., Lemeshow S. Sample Size Determination in Health Studies- A practical Manual. 1st Edition. Geneva, Switzerland: World Health Organization; 1991.

[25] CDC; Centre for Disease Communication. Ntaional Health and Examination Survey (NHANES): Anthropometry Procedures Manual. 2009.

[26] WHO; World Health Organization. Global Database on Body Mass Index; BMI classification. Geneva, Switzerland: 2006.

[27] WHO; World Health Organization. Waist Circumference and Waist-Hip Ratio Report of a WHO Expert Consultation. Geneva, Switzerland: 2008.

[28] WHO; World Health Organization. Global Physical Activity Questionnaire (GPAQ) WHO STEPwise approach to NCD risk factor surveillance. n.d.

[29] WHO; World Health Organization. Global Physical Activity Questionnaire (GPAQ) Analysis Guide. Geneva, Switzerland: 2010. doi: 10.1016/S0140-6736(12)60736-3.

[30] Bucolo G, David H. Quantitative Determination of Serum Triglycerides by the Use of Enzymes. Clin Chem 1973; 19: 476-82.

[31] Allain CC, Poon LS, Chan CSG, Richmond W, Fu PC. EnzymaticDetermination of Total Serum Cholesterol. Clin Chem 1974; 20: 470-5.

[32] Keppy NK, Bain G, Ph D, Allen MW, Scientific TF. Enzymatic Colorimetric Methods for the Analysis of Human Serum by UV-Visible Spectroscopy. Madison, WI, USA: n.d.

[33] Assmann G, Schriewer H, Schmltz G, Edgar-otto H. Quantification of High-Density-Lipoprotein Cholesterol by Precipitation with Phosphotungstic Acid / MgCI2. Clin Chem 1983; 29: 2026-30.

[34] Friedewald WT, Levy RI, Fredrickson DS. Estimation of the concentration of Low-Density Lipoprotein in Plasma, Without use of the Preparative Ultracentrifuge. Clin Chem 1972; 18: 499-502.

[35] Klenk DC, Hermanson GT, Krohn RI, Smith PK, England JD, Fujimoto K, et al. Determination of Glycosylated Hemoglobin by Affinity Chromatography: Comparison with Colorimetric and Ion-Exchange Methods, and Effects of Common Interferences. Clin Chem 1982; 28: 2088-94.

[36] Beach EF, Turner JJ. An Enzymatic Method for Glucose Determination in Body Fluids 1958; 4: 462-75.

[37] American Diabetes Association. Standards of Medical Care in. J Clin Appl Res Educ 2017; 36: 5-8. doi: 10.2337/dc13-S011. 
[38] AAC; American College of Cardiology. 2017 Guideline for the Prevention, Detection, Evaluation, and Management of High Blood Pressure in Adults. 2017. doi: 10.1016/j.jacc.2017.07.745.

[39] Davidson JA, Einhorn D, Ganda OP, Garber AJ, Jellinger PS, Jovanovič L, et al. HHS Public Access 2016; 21. doi: 10.4158/EP15672.GL.To.

[40] AACE; American Association of Clinical Endocrinologists and ACE; American College of Endocrinology. Consensus Statement by the American Association of Clinical Endocrinologists and American College of Endocrinology on the Comprehensive Type 2 Diabetes Management Algorithm 2017 Executive Summary. Endocr Pract 2017; 23: 207-38. doi: 10.4158/EP161682.CS.

[41] Unadike, BC; Akpan, NA; Peters, EJ; Essien IEO. Prevalence of the Metabolic Syndrome among Patients. African J Endocrinol Metab 2009; 8: 7-9.

[42] Otero LM, Zanetti ML, Tiexeira CR de S. Sociodemographic and clinical characteristics of a diabetic population 1 at a primary level health care center 2007; 15: 768-73.

[43] Alwan AH, Alhusuny A, Ali A, Alwan H, Medicine I, B EMBC. Assessment of Metabolic Syndrome and Its Risk Factors among Patients with Type 2 DM at Merjan Teaching Hospital, Al-Hilla City. J Dent Med Sci 2014; 13: 69-75.

[44] Patel JL, Suthar AM, Dalsaniya VB, Parikh AP, Suthar NN, Patel KL. A Study of Metabolic Syndrome and its and their Asymptomatic First-degree Relatives. Indian J Clin Pract 2013; 23: 520-33.

[45] Raman R, Aditi G, Swakshyar S, Pal Suganeswari G, Kadri, Venkatesh Vaitheeswaran, Kulothungan Tarun S, Access O. Prevalence of Metabolic Syndrome and its influence on microvascular complications in the Indian population with Type 2 Diabetes Mellitus. Sankara Nethralaya Diabetic Retinopathy Epidemiology And Molecular Genetic Study. Diabetol Metab Syndr 2010; 2: 1-9. doi: 10.1186/1758-59962-67.

[46] Moreira SS, Andrade CDS, Antonio C, Teles DS, Neves RCS. Correlates of poor glycemic control in patients with type 2 diabetes: results of a nationwide survey in Brazil. Diabetol Metab Syndr 2015; 7: A55. doi: 10.1186/1758-5996-7-S1A55.

[47] Wallace TM, Matthews DR. Poor glycaemic control in type 2 diabetes: a conspiracy of disease, suboptimal therapy and attitude. J Med 2000: 369-74.
[48] Tamara B. Horwich GCF. Glucose, Obesity, Metabolic Syndrome, and Diabetes: Relevance to Incidence of Heart Failure. J Am Coll Cardiol 2010; 55: 283-93. doi: 10.1016/j.jacc.2009.07.029. Glucose.

[49] Rodrigues TC, Canani LH, Gross JL. Review Article Metabolic Syndrome, Insulin Resistance and Cardiovascular Disease in Type-1 Diabetes Mellitus. Cardiovasc Dis Type-1 Diabetes Mellit 2008: 125-30.

[50] Hu H, Hori A, Nishiura C, Sasaki N, Okazaki H. Hbalc, Blood Pressure, and Lipid Control in People with Diabetes: Japan Epidemiology Collaboration on Occupational Health Study. PLoS One 2016; 11: 1-13. doi: 10.1371/journal.pone.0159071.

[51] Godwill EA, Okolie A, Nwalo F, Akaniro-ejim NE. Association of Obesity and Dyslipidaemia with Type 2 Diabetes in Outpatients of Enugu State University Teaching Hospital (ESUTH) in Enugu Nigeria. J Life Sci 2018; 12. doi: 10.17265/1934-7391/2018.02.003.

[52] Firouzi S, Nisak B, Yusof M, Kamaruddin NA. Nutritional status, glycemic control and its associated risk factors among a sample of type 2 diabetic individuals, a pilot study 2015 .

[53] Tadewos A, Ambachew H, Assegu D. Pattern of Metabolic Syndrome in Relation to Gender among Type-II DM Patients in Hawassa University Comprehensive Specialized Hospital, Hawassa,. Heaalth Sci J 2017; 11: 509. doi: 10.21767/1791809X.1000509.

[54] Mavarez-Martinez A, Gonzalez-Zacarias AA, Stoicea N, Rogers B, Arias-Morales CE. Impact of Demographic, Socioeconomic, and Psychological Factors on Glycemic SelfManagement in Adults with Type 2 Diabetes Mellitus. Front Public Heal 2016; 4: 1-8. doi: 10.3389/fpubh.2016.00195.

[55] Barlin H, Mercan M. Occupation and Obesity: Effect of Working Hours on Obesity by Occupation Occupation and Obesity: Effect of Working Hours on Obesity by Occupation Groups 2016. doi: 10.11114/aef.v3i2.1351.

[56] Ogunsina K, Dibaba DT, Akinyemiju T. Status and prevalence of cardio-metabolic risk factors in five middle-income countries 2018; 8: 1-10. doi: 10.7189/jogh.08.020405.

[57] Gopalakrishnan S, Geetha A, R U. Study on the impact of family history of diabetes among type 2 diabetes mellitus patients in an urban area of Kancheepuram district, Tamil Nadu. Int J Community Med Public Heal 2017; 4. doi: 10.18203/2394-6040.ijcmph20174819. 